\title{
Microtubules in Dendritic Spine Development
}

\author{
Jiaping Gu, ${ }^{1}$ Bonnie L. Firestein, ${ }^{2}$ and James $Q$. Zheng ${ }^{1}$ \\ ${ }^{1}$ Department of Neuroscience and Cell Biology, University of Medicine and Dentistry of New Jersey, Robert Wood Johnson Medical School, Piscataway, New \\ Jersey 08854, and 2Department of Cell Biology and Neuroscience, Rutgers University, Piscataway, New Jersey 08854
}

It is generally believed that only the actin cytoskeleton resides in dendritic spines and controls spine morphology and plasticity. Here, we report that microtubules (MTs) are present in spines and that shRNA knockdown of the MT plus-end-binding protein EB3 significantly reduces spine formation. Furthermore, stabilization and inhibition of MTs by low doses of taxol and nocodazole enhance and impair spine formation elicited by BDNF (brain-derived neurotrophic factor), respectively. Therefore, MTs play an important role in the control and regulation of dendritic spine development and plasticity.

Key words: cytoskeleton; hippocampus; plasticity; synapse; neurotrophin; synaptic plasticity

\section{Introduction}

A majority of excitatory synapses reside on dendritic spines, tiny protrusions that serve as a platform for postsynaptic specializations as exemplified by clustered neurotransmitter receptors at the postsynaptic density (PSD). Changes in postsynaptic structures, particularly spines, are considered a hallmark of synaptic modification (Yuste and Bonhoeffer, 2001; Segal, 2005) that underlies developmental refinement of neural circuitry, learning and memory, aging, and neurodegenerative diseases (Geinisman, 2000; Yuste and Bonhoeffer, 2001; Nimchinsky et al., 2002; Halpain et al., 2005). It is well established that the actin cytoskeleton is the major cytoskeletal structure that controls and regulates spine formation and dynamics (Tada and Sheng, 2006). Live-cell imaging confirms that changes in the size or shape of a spine are linked to changes in its actin cytoskeleton (Fukazawa et al., 2003; Okamoto et al., 2004; Calabrese et al., 2006). Recent studies have also demonstrated in culture and in vivo that actin polymerization is coupled to spine enlargement during long term potentiation (Fukazawa et al., 2003; Okamoto et al., 2004), whereas long term depression involves spine shrinkage through actin depolymerization (Okamoto et al., 2004; Zhou et al., 2004).

In contrast, the presence of MTs and associated proteins in spines and their role in spine plasticity remain underexplored. Early electron microscopy studies suggest that a highly labile population of MTs exists within dendritic spines in close proximity to the PSD (Westrum et al., 1980; Chicurel and Harris, 1992). In addition, tubulin mRNA has been found in synaptosomal preparations enriched for dendritic spines (Chicurel et al., 1993), and $\beta$-tubulin was found in the PSD by biochemical methods (Kelly

Received June 3, 2008; revised Sept. 9, 2008; accepted Sept. 22, 2008.

This work was supported by National Institutes of Health GM083889 and AG029596 (J.Q.Z.), and National Science Foundation Grant 0548543 and March of Dimes Foundation Grant 1-FY04-107 (B.L.F.). We thank Dr. Anna Akhmanova (Department of Cell Biology, Erasmus Medical Center, 3000 CA Rotterdam, The Netherlands) for sharing with us the shRNA-EB3 construct and the anti-EB3 antibody. We are also grateful for technical assistance from Danie Komlos (Rutgers) on mOrange subcloning and phalloidin staining of spines.

Correspondence should be addressed to James Q. Zheng at his present address, Department of Cell Biology, Emory University School of Medicine, 615 Michael Street, Atlanta, GA 30322. E-mail: james.zheng@emory.edu. D0I:10.1523/JNEUROSCI.2509-08.2008

Copyright $\odot 2008$ Society for Neuroscience $\quad$ 0270-6474/08/2812120-05\$15.00/0 and Cotman, 1978; Li et al., 2004) and immunocytochemistry (Caceres et al., 1983). The physical interaction between soluble forms of tubulin, such as tubulin heterodimers, and postsynaptic proteins, such as NMDA receptor (NMDAR) subunits, has also been demonstrated (van Rossum et al., 1999). Other indirect evidence comes from the existence of microtubule associated protein 2 in the postsynaptic region, and that activation of NMDAR can decrease its phosphorylation (Quinlan and Halpain, 1996). Despite these early findings, MTs are not considered to enter spines nor do they play a role in spine development and dynamics. Such a notion, however, might be attributable to limited imaging techniques for capturing a small number of dynamic MTs entering the tiny actin-rich protrusions. Recent reports show the presence of microtubule structures in spines under certain conditions, such as strong tetanic stimulation to induce long-term potentiation (Mitsuyama et al., 2008), or during recovery from slice preparation (Fiala et al., 2003). It is thus conceivable to speculate that transient polymerization of microtubules in spines might contribute to spine regulation during plasticity (van Rossum and Hanisch, 1999). Using live confocal imaging, we show here that MTs are present in dendritic spines. Furthermore, we find that knockdown of the MT plus-endbinding protein EB3 impairs spine formation. Finally, manipulation of MT dynamics by specific pharmacological drugs modulates spine development induced by brain derived neurotrophic factor. These results thus indicate an important role for microtubules in the formation and plasticity of dendritic spines.

\section{Materials and Methods}

Constructs and reagents. pEGFP-C1, pmOrange, and pEGFP-Tub constructs were from Clontech. The mOrange coding sequence was subcloned into pEGFP-N1 vector [with green fluorescent protein (GFP) sequence excised] to allow expression in mammalian cells. Hairpin constructs encoding RNAi to EB3 were inserted into pEGFP-C1 vector (Komarova et al., 2005). Recombinant human brain-derived neurotrophic factor (BDNF) was purchased from Peprotech, and taxol and nocodazole were from Sigma. Neuronal class III $\beta$-tubulin antibodies were from Covance.

Hippocampal cultures, transfection, and imaging. Hippocampal neurons were prepared from hippocampal tissue of rat embryos (embryonic 


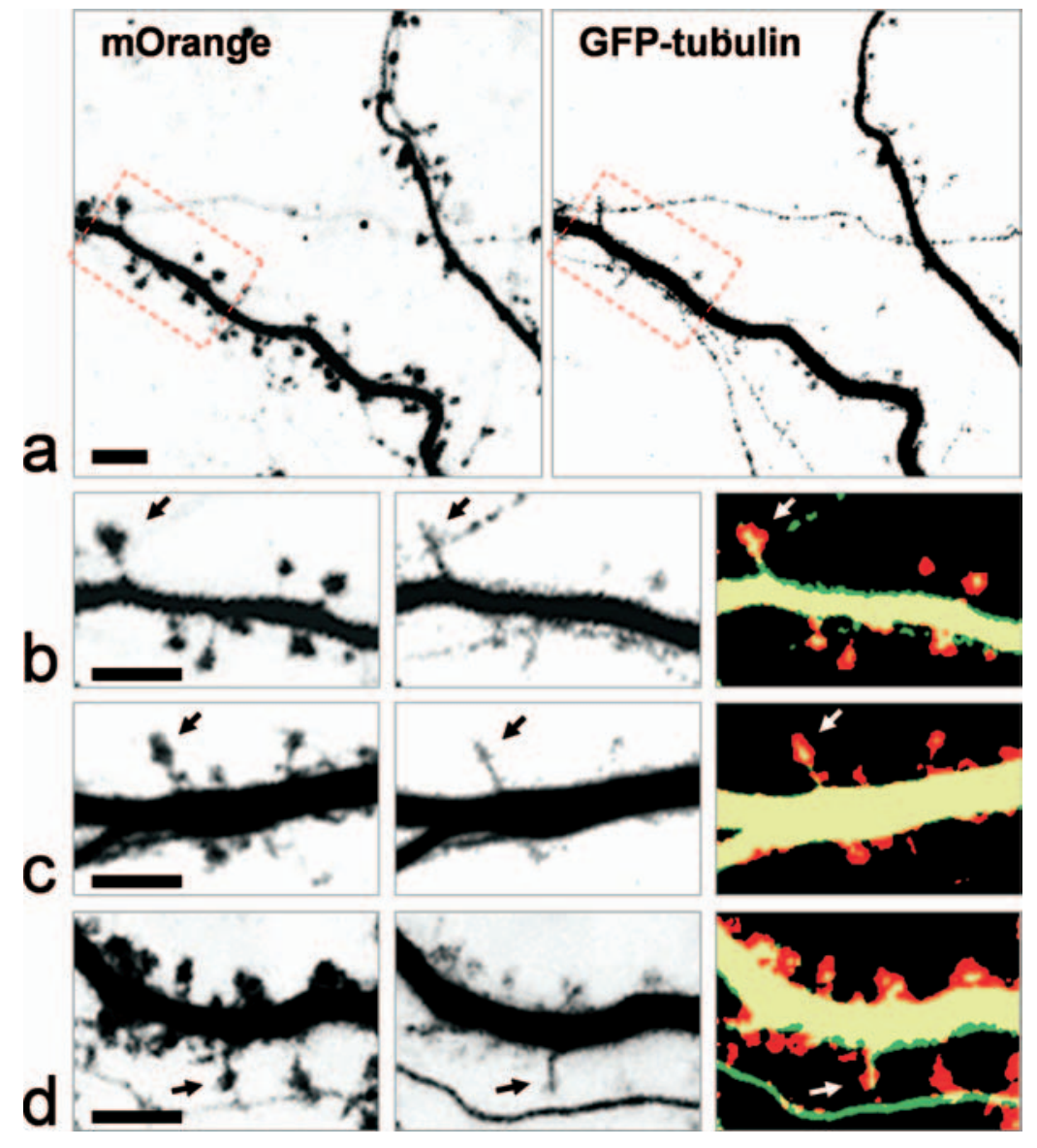

Figure 1. Confocal live imaging of dendritic spines and microtubules. Cultured hippocampal neurons were transfected with pm0range and pEGFP-tubulin. Dual-channel imaging was achieved using the frame-channel acquisition mode to eliminate bleed through of two fluorophores. $\boldsymbol{a}$, Representative images of the dendritic processes of a hippocampal neuron at DIV21. The spines are highlighted by m0range fluorescence (left), and microtubules by GFP-tubulin (right). $\boldsymbol{b}$, The region outlined by dashed rectangles is shown at higher magnification. Arrows indicate a mushroom-shaped spine that contains clear microtubules. $\boldsymbol{c}, \boldsymbol{d}$, Representative images of spines from different neurons showing the presence of MTs in spines. Scale bar, $5 \mu \mathrm{m}$.

number of spines of all types per unit length of parent dendrite, and normalizing to $1 \mu \mathrm{m}$ of length. For BDNF and MT-drug experiments, the same region of the neuron was repeatedly imaged and compared before and after treatment. At least three rounds of cultures were used in each experiment with $>300$ spines measured.

Immunofluorescent staining. Neurons were simultaneously fixed and permeabilized for 30 $\min$ at $37^{\circ} \mathrm{C}$ in $4 \%$ paraformaldehye in PHEM buffer (60 mM PIPES, $25 \mathrm{~mm}$ HEPES, $10 \mathrm{~mm}$ EGTA, $2 \mathrm{~mm} \mathrm{MgCl}_{2}, 0.12 \mathrm{M}$ sucrose, $\mathrm{pH}$ 7.0) supplemented with $0.25 \%$ glutaraldehyde, 10 $\mu \mathrm{M}$ taxol, and $0.1 \%$ Triton X-100 (Dent and Kalil, 2001) After 3 rinses in PBS, cells were blocked with $10 \%$ BSA in PBS, labeled with primary and secondary antibodies. Then a low concentration of rhodamine-phalloidin (Invitrogen; 1:1000 of the stock) was used to label actin filaments. Images were acquired by the Nikon C1 confocal system.

\section{Results}

Isolated hippocampal neurons cultured at high density for 2 weeks were used for the following experiments. These neurons form extensive synaptic connections over 2 weeks and display numerous dendritic spines. While spines from two-week old cultures vary in morphology, ranging from thin and long filopodia to mushroom-shaped "mature" spines, a large percentage of spines become mushroom-shaped after 3 weeks in culture. To image spines and microtubules, we coexpressed mOrange and GFP- $\alpha$ tubulin in hippocampal neurons via transfection of plasmids using the calcium phosphate method. The transfection efficiency was controlled at a low level (1-5\%)

day 18) according to the method previously described (Banker and Cowan, 1977). The calcium phosphate method was used to transfect cultured hippocampal neurons (Köhrmann et al., 1999). Images were acquired using a Nikon C1 laser scanning confocal system on a Nikon microscope. Cultured neurons were changed from culture medium to Kreb's-Ringer's saline [(in mM) $150 \mathrm{NaCl}, 5 \mathrm{KCl}, 2 \mathrm{CaCl}_{2}, 1 \mathrm{MgCl}_{2}, 10$ glucose, 10 HEPES, pH 7.4] (Bacci et al., 1999) for imaging. The original culture medium was added back into cell culture after each imaging. Neurons were examined daily for up to 1 week for long-term recording of spine development and dynamics. BDNF and MT drugs were added to the bath medium after the first day imaging and supplemented to the medium daily afterward. To image the same neuron over a long period, a low resolution picture of the cell and several high resolution pictures of dendritic regions were taken, and the approximate location in dish was recorded separately, both of which were used to locate the same neuron on the next day.

Quantitative analysis of spine number and morphology. Dendritic protrusions were divided into two categories: spines (length, $<3 \mu \mathrm{m}$ ) and filopodia $(3 \sim 10 \mu \mathrm{m})$ (Tyler and Pozzo-Miller, 2003). Spine numbers were counted manually. The length and width of spines were measured using ImageJ. For mushroom and thin spines, the maximal width of spine head was measured, whereas for stubby spines, the width at halfway between the tip and the base of spine was taken. To measure the intensity profile of the spine, we used ImageJ to draw a line across the spine at where the width was measured. Intensity profiles of both channels (red: mOrange; green: GFP-tubulin) were then generated using the Image line profile function. Spine density was calculated by quantifying the to allow a close examination of the dendritic spines of one neuron over time, without interference from others. Neurons were transfected 2-3 d before imaging. Frame-channel acquisition of a three-dimensional (3-D) stack of the dendritic regions was performed, followed by volume rendering of the dendrites and associated spines, thus minimizing the possibility of missing some parts of the tiny spines from optical sectioning. Over many rounds of imaging, we consistently observed a significant number of dendritic spines that contain microtubules (Fig. 1, supplemental Fig. 1, available at www.jneurosci.org as supplemental material). This is much more apparent for mushroom-shaped spines, since the GFP-tubulin signals are clearly restricted inside the spine head with an appearance of polymerized MTs (Fig. $1 b-d$, arrows).

To further analyze spines containing MTs, we examined the fluorescent intensity profiles of both mOrange and GFP signals for each spine containing GFP-tubulin signals (Fig. 2). Based on their morphologies, spines were categorized into three groups for this analysis: mushroom-shaped, filopodia-like, and stubby. In many mushroom spines, there is a clear difference between intensity profiles of mOrange (red) and GFP-tubulin (green), indicating good separation of two fluorescent channels and the likelihood of polymerized MTs in spines. We also observed filopodia and thin spines containing strong GFP-tubulin signals, but in 
most cases, the two fluorescent profiles overlap, making it difficult to determine whether the GFP-tubulin signals represent soluble tubulin proteins or polymerized MTs. We calculated the percentage of spines containing GFP-tubulin for each spine group and found that filopodia-like spines have a higher percentage containing GFP-tubulin (Fig. 2). Because only mushroom spines generated distinct intensity profiles of the spine (depicted

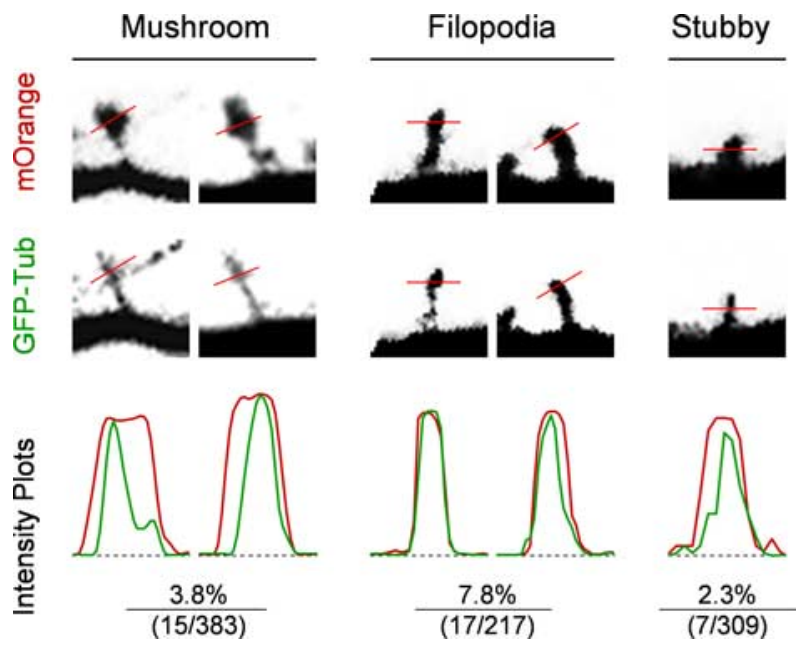

Figure 2. Intensity profile analyses of spines containing GFP-tubulin signals. Live confocal images of both m0range and GFP-tubulin were subjected to line profile analyses. Dendritic spines were categorized into three groups: mushroom-shape, filopodia-like, and stubby. A line was drawn across the spine (at the widest part of spine for mushroom and filopodia spines, and at the halfway from dendrite to spine tip for stubby spines), and the intensity profiles of m0range (red) and GFP (green) signals are shown. Only the mushroom-shaped spines exhibit a clear difference in the intensity profiles between the red and green channels, indicating that they likely contain microtubules rather than diffuse tubulin monomers and dimers. The percentages of each type of spines containing GFP-tubulin signals, together with the raw numbers (number containing GFP-tubulin/total number of spines), are shown. Data were collected from cultured hippocampal neurons (DIV21) from at least three independent groups of experiments.
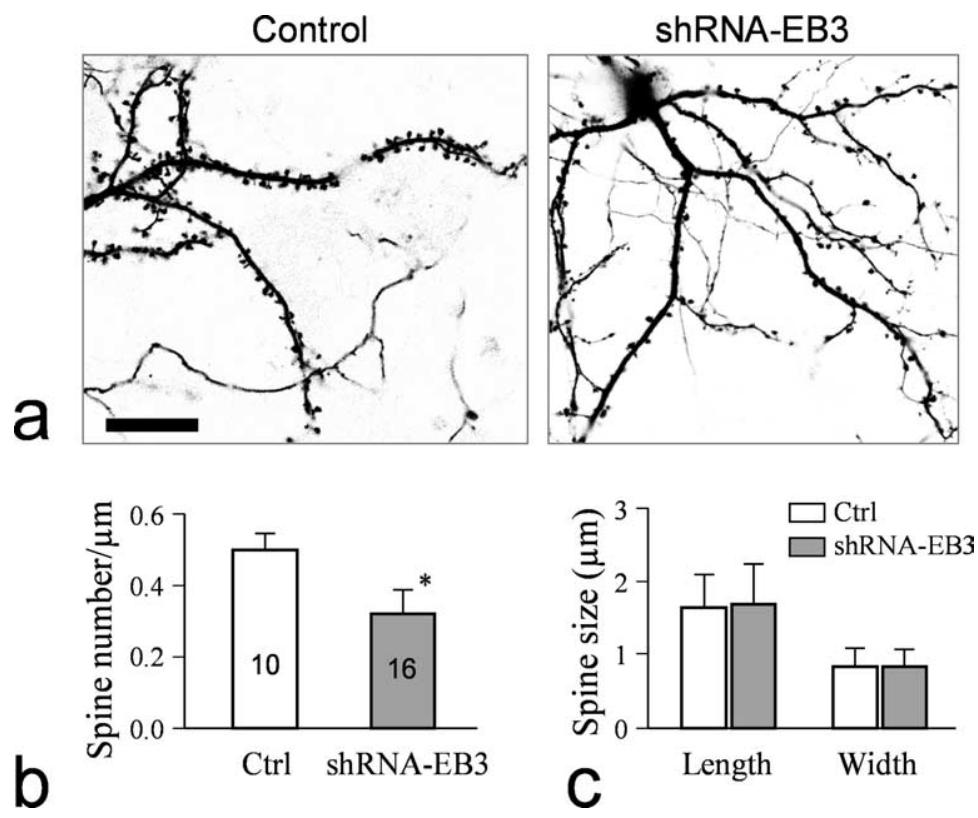

Figure 3. Knockdown of EB3 by shRNA reduces the number of dendritic spines. $\boldsymbol{a}$, Representative images of dendritic processes and spines in a control neuron expressing GFP and a neuron coexpressing shRNA-EB3 and GFP. Scale bar, $20 \mu \mathrm{m}$. $\boldsymbol{b}$, Quantification of the spine numbers for the control and shRNA-EB3 expressing neurons. Asterisk indicates a statistical significance ( $p<0.05$, Student's $t$ test). c, Measurements of the spine size (width and length). by mOrange fluorescence) and MTs (indicated by GFP-tubulin signals), we therefore only counted those mushroom spines containing MTs, which represent $1.6 \%$ of all of the spines (three groups) examined. Such a small but significant percentage is in part attributable to our stringent criteria for determining spines containing MTs. It is also likely that the low percentage reflects the transient nature of microtubules entering spines. Nevertheless, our 3-D live imaging data present the clear visual evidence for the presence of MTs in dendritic spines. It should be noted that the observed presence of MTs in spines is unlikely an artifact of GFP-tubulin expression, because our immunostaining using antibodies against endogenous tubulin also revealed the presence of MTs in dendritic spines (supplemental Fig. 2, available at www.jneurosci.org as supplemental material).

Recent studies have shown that the plus ends of MTs are decorated with a variety of proteins including the EB1 family of the plus-end-tracking proteins (+TIPs) that regulate MT dynamics and interactions with other cellular components (Akhmanova and Hoogenraad, 2005). One member of the EB1 family, EB3, is preferentially expressed in the nervous system (Nakagawa et al., 2000). We found that EB3 is widely expressed in hippocampal neuron cultures, including dendrites and spines (supplemental Fig. 3, available at www.jneurosci.org as supplemental material). To explore the role of MTs in spines, we used shRNA to knock down EB3. This shRNA-EB3 has been used to effectively knock down EB3 in cultured cells (Komarova et al., 2005). To examine the effect of EB3 knockdown on spine development, we transfected 2-week-old hippocampal cultures and examined spines 1 week later. Our data show that EB3 knockdown significantly reduced the number of spines in cultured hippocampal neurons (Fig. 3a). Compared with the parallel control group, EB3 knockdown reduced the number of spines to $\sim 60 \%$ of the control neurons (Fig. $3 b$ ), without significantly altering the spine length and width (Fig. 3c). These data indicate the importance of MTplus-end proteins in spine development.

BDNF has been shown to potentiate synaptic connections through its presynaptic and postsynaptic actions (Lu, 2004). Using live confocal imaging to monitor the same GFP-expressing neurons over time, we examined the effects of BDNF on spine development. High-resolution imaging of GFPexpressing neurons and their dendritic branches and spines was performed daily after transfection. We examined the changes in spine formation in the same dendritic regions at different days after BDNF exposure, thus eliminating potential artifacts from variations among different neurons and different dendritic regions. We found that $24 \mathrm{~h}$ exposure of neurons to BDNF markedly increased the number of spines along the dendritic processes (Fig. $4 b$ ) compared with the control group (Fig. 4a). Quantification of spine numbers along dendritic processes indicates that $24 \mathrm{~h}$ treatment with BDNF increased the number of spines to $\sim 120 \%$ of the control group (Fig. 4d). BDNFpromoted spine formation was mostly observed in hippocampal neurons cultured for 2 weeks, whereas 3-week-old cultures did not show significant changes (Fig. $4 d$ ). 

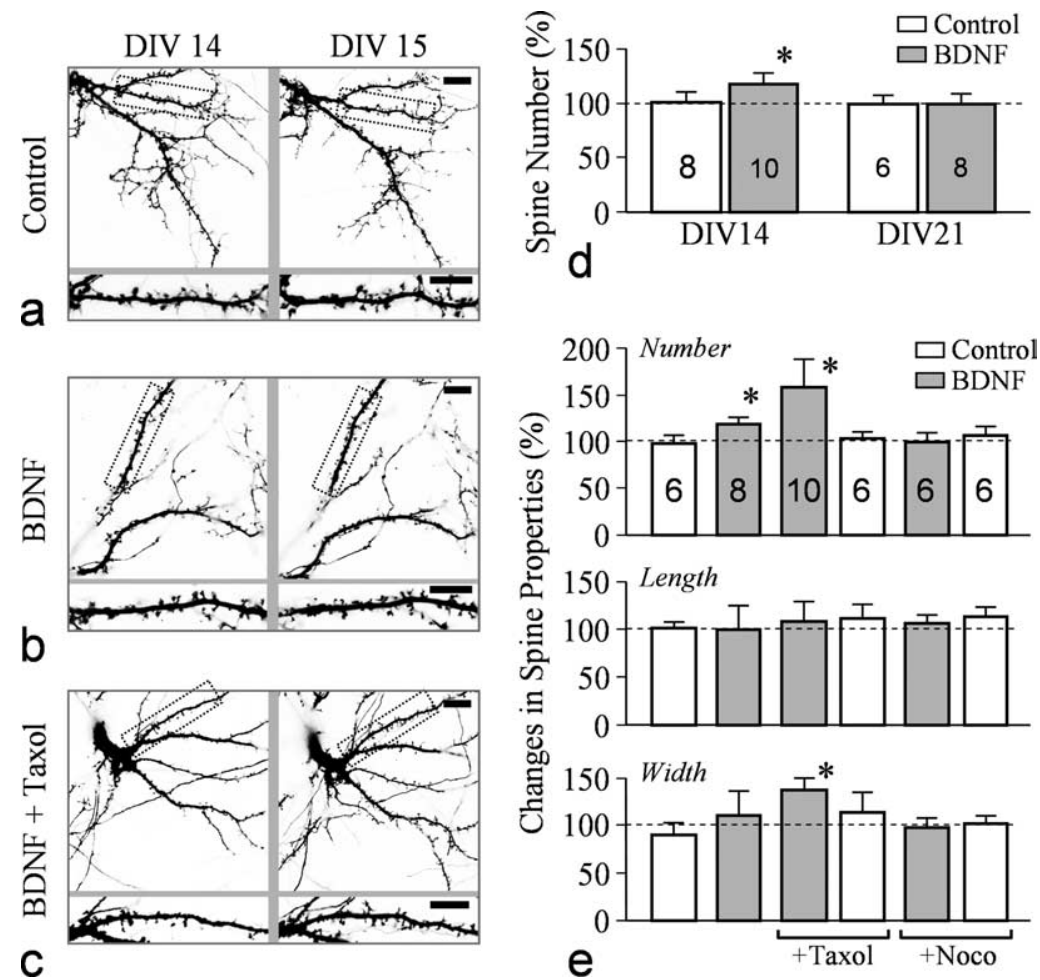

Figure 4. BDNF-elicited spine formation is enhanced by taxol. $\boldsymbol{a}$, Representative images of dendritic processes and spines in a GFP-expressing neuron at DIV14 and DIV15. A portion of the dendrite (dotted rectangles) is enlarged to show spines (bottom row). $\boldsymbol{b}$, Representative images showing the dendritic processes and spines of a neuron before and after $24 \mathrm{~h}$ exposure to BDNF. $\boldsymbol{c}$, Representative images showing the dendritic processes and spines of a neuron before and after $24 \mathrm{~h}$ exposure to BDNF $(50 \mathrm{ng} / \mathrm{ml})$ and taxol ( $10 \mathrm{~nm})$. Scale bars: $\boldsymbol{a}-\boldsymbol{c}, 10 \mu \mathrm{m} . \boldsymbol{d}$, Quantitative analysis of the increase in spine numbers in control and BDNF-treated groups from DIV14 and DIV21 cultures. The asterisk indicates statistical significance when comparing the BDNF group to the

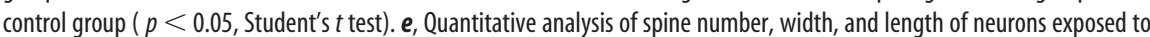
BDNF together with different MT-altering drugs. Nocodazole (Noco) was at $500 \mathrm{~nm}$. Asterisks indicate statistical significance when compared with the control group ( $p<0.05$, Student's $t$ test).

To examine the functional role of MTs in BDNF function, we applied a low concentration of taxol and nocodazole to hippocampal neurons and examined their effects on BDNF-induced spine formation. Low concentrations of taxol are known to promote and stabilize MTs, whereas low doses of nocodazole impede the dynamic properties of the MT $(+)$ ends without causing disassembly (Buck and Zheng, 2002). Interestingly, we found that the presence of taxol $(10 \mathrm{nM})$ further increased spine formation in the presence of BDNF (Fig. 4c). Quantification of spine numbers shows that the presence of taxol further enhanced BDNFinduced spine formation to $\sim 150 \%$ of the control group, whereas $500 \mathrm{~nm}$ nocodazole blocked the effect of BDNF (Fig. 4e). Moreover, combining taxol with BDNF increased spine width without changing spine length, indicating larger and more "mature" spines. Application of either of these MT-altering drugs alone did not affect baseline spine formation for a period of $24 \mathrm{~h}$. Together, these findings provide further evidence that MTs play an important role in spine development.

\section{Discussion}

Dendritic spines are known for their high levels of actin and actin-associated proteins, which are believed to control and regulate spine morphology and dynamics associated with neural development and activity-dependent plasticity (Tada and Sheng, 2006). In contrast, MTs have been ignored for playing a role in dendritic spine formation and plasticity because of the fact that no reliable imaging data have shown the presence of MTs in spines. In this study, we used live 3-D confocal imaging to show the presence of MTs in spines, especially in mushroom-shaped "mature spines." The small percentage of spines containing MTs may reflect the transient and dynamic nature of these MTs entering spines. Indeed, a large number of spines were found to be explored by MTs, but the time MTs stayed in each spine was limited to a few minutes [Dr. Erik Dent (University of Wisconsin, Madison, WI) personal communication]. In addition to imaging data, we show that knockdown of the + TIP protein EB3 impairs spine formation, and pharmacological manipulation of MT dynamics affects spine formation elicited by BDNF. It is of particular interest to note that a low concentration of taxol was able to further enhance spine formation induced by BDNF. Previous studies on nerve growth cones have shown that local stabilization of MTs by low doses of taxol promotes actin-based lamellipodial protrusion and growth cone attraction, which might involve MT activation of Rac1 GTPases (Buck and Zheng, 2002). It is plausible that the low doses of taxol promote spine protrusions induced by BDNF through MT stabilization. Alternatively, low doses of taxol may prevent the elimination/shrinkage of some spines, leading to a net increase in spine numbers.

It should be noted that, at this moment, we cannot rule out the possibility that both EB3 knockdown and MT drug treatment might grossly affect MTs and their functions (e.g., transport of cargo) in dendritic shafts and/or entire neurons, which would in turn affect spine development and plasticity. Although the low concentrations of MT drugs used here have been shown to mainly affect the dynamic MT tips without grossly disrupting MT structures (Buck and Zheng, 2002), it is plausible that some MT functions in dendritic shafts could be affected, thus influencing spine development. Future experiments using spatially restricted application of MT drugs (e.g., photouncaging) to a selected number of spines would enable one to address this important question. Nonetheless, our results indicate an important role for MTs in spine development and dynamics. Dynamic MTs exploring spines may be responsible for many cellular aspects of spine development, including the conversion of dendritic filopodia to spines, delivery of membranous cargos to spines, and interactions with the actin cytoskeleton for spine dynamics. Future studies are clearly required to elucidate the functions and mechanisms of dynamic MTs in regulating spine development.

\section{References}

Akhmanova A, Hoogenraad CC (2005) Microtubule plus-end-tracking proteins: mechanisms and functions. Curr Opin Cell Biol 17:47-54.

Bacci A, Verderio C, Pravettoni E, Matteoli M (1999) Synaptic and intrinsic mechanisms shape synchronous oscillations in hippocampal neurons in culture. Eur J Neurosci 11:389-397.

Banker GA, Cowan WM (1977) Rat hippocampal neurons in dispersed cell culture. Brain Res 126:397-425. 
Buck KB, Zheng JQ (2002) Growth cone turning induced by direct local modification of microtubule dynamics. J Neurosci 22:9358-9367.

Caceres A, Payne MR, Binder LI, Steward O (1983) Immunocytochemical localization of actin and microtubule-associated protein MAP2 in dendritic spines. Proc Natl Acad Sci U S A 80:1738-1742.

Calabrese B, Wilson MS, Halpain S (2006) Development and regulation of dendritic spine synapses. Physiology (Bethesda) 21:38-47.

Chicurel ME, Harris KM (1992) Three-dimensional analysis of the structure and composition of CA3 branched dendritic spines and their synaptic relationships with mossy fiber boutons in the rat hippocampus. J Comp Neurol 325:169-182.

Chicurel ME, Terrian DM, Potter H (1993) mRNA at the synapse: analysis of a synaptosomal preparation enriched in hippocampal dendritic spines. J Neurosci 13:4054-4063.

Dent EW, Kalil K (2001) Axon branching requires interactions between dynamic microtubules and actin filaments. J Neurosci 21:9757-9769.

Fiala JC, Kirov SA, Feinberg MD, Petrak LJ, George P, Goddard CA, Harris KM (2003) Timing of neuronal and glial ultrastructure disruption during brain slice preparation and recovery in vitro. J Comp Neurol 465:90-103.

Fukazawa Y, Saitoh Y, Ozawa F, Ohta Y, Mizuno K, Inokuchi K (2003) Hippocampal LTP is accompanied by enhanced F-actin content within the dendritic spine that is essential for late LTP maintenance in vivo. Neuron 38:447-460.

Geinisman Y (2000) Structural synaptic modifications associated with hippocampal LTP and behavioral learning. Cereb Cortex 10:952-962.

Halpain S, Spencer K, Graber S (2005) Dynamics and pathology of dendritic spines. Prog Brain Res 147:29-37.

Kelly PT, Cotman CW (1978) Synaptic proteins. Characterization of tubulin and actin and identification of a distinct postsynaptic density polypeptide. J Cell Biol 79:173-183.

Köhrmann M, Haubensak W, Hemraj I, Kaether C, Lessmann VJ, Kiebler MA (1999) Fast, convenient, and effective method to transiently transfect primary hippocampal neurons. J Neurosci Res 58:831-835.

Komarova Y, Lansbergen G, Galjart N, Grosveld F, Borisy GG, Akhmanova A (2005) EB1 and EB3 control CLIP dissociation from the ends of growing microtubules. Mol Biol Cell 16:5334-5345.

Li KW, Hornshaw MP, Van Der Schors RC, Watson R, Tate S, Casetta B, Jimenez CR, Gouwenberg Y, Gundelfinger ED, Smalla KH, Smit AB (2004) Proteomics analysis of rat brain postsynaptic density. Implications of the diverse protein functional groups for the integration of synaptic physiology. J Biol Chem 279:987-1002.
Lu B (2004) Acute and long-term synaptic modulation by neurotrophins. Prog Brain Res 146:137-150.

Mitsuyama F, Niimi G, Kato K, Hirosawa K, Mikoshiba K, Okuya M, Karagiozov K, Kato Y, Kanno T, Sanoe H, Koide T (2008) Redistribution of microtubules in dendrites of hippocampal CAl neurons after tetanic stimulation during long-term potentiation. Ital J Anat Embryol 113:17-27.

Nakagawa H, Koyama K, Murata Y, Morito M, Akiyama T, Nakamura Y (2000) EB3, a novel member of the EB1 family preferentially expressed in the central nervous system, binds to a CNS-specific APC homologue. Oncogene 19:210-216.

Nimchinsky EA, Sabatini BL, Svoboda K (2002) Structure and function of dendritic spines. Annu Rev Physiol 64:313-353.

Okamoto K, Nagai T, Miyawaki A, Hayashi Y (2004) Rapid and persistent modulation of actin dynamics regulates postsynaptic reorganization underlying bidirectional plasticity. Nat Neurosci 7:1104-1112.

Quinlan EM, Halpain S (1996) Postsynaptic mechanisms for bidirectional control of MAP2 phosphorylation by glutamate receptors. Neuron $16: 357-368$.

Segal M (2005) Dendritic spines and long-term plasticity. Nat Rev Neurosci 6:277-284.

Tada T, Sheng M (2006) Molecular mechanisms of dendritic spine morphogenesis. Curr Opin Neurobiol 16:95-101.

Tyler WJ, Pozzo-Miller L (2003) Miniature synaptic transmission and BDNF modulate dendritic spine growth and form in rat CA1 neurones. J Physiol 553:497-509.

van Rossum D, Hanisch UK (1999) Cytoskeletal dynamics in dendritic spines: direct modulation by glutamate receptors? Trends Neurosci 22:290-295.

van Rossum D, Kuhse J, Betz H (1999) Dynamic interaction between soluble tubulin and C-terminal domains of N-methyl-D-aspartate receptor subunits. J Neurochem 72:962-973.

Westrum LE, Jones DH, Gray EG, Barron J (1980) Microtubules, dendritic spines and spine appratuses. Cell Tissue Res 208:171-181.

Yuste R, Bonhoeffer T (2001) Morphological changes in dendritic spines associated with long-term synaptic plasticity. Annu Rev Neurosci 24:1071-1089.

Zhou Q, Homma KJ, Poo MM (2004) Shrinkage of dendritic spines associated with long-term depression of hippocampal synapses. Neuron 44: 749-757. 